Gazi University
Journal of Science
$\mathrm{http} / / /$ dergipark.gov.tr/gujs

\title{
Polynomial Parametric Equations of Rectifying Salkowski Curves
}

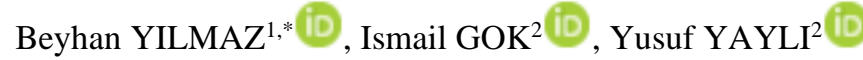 \\ ${ }^{1}$ Kahramanmaraş Sütçü Imam University, Faculty of Science, Department of Mathematics, 46100, Kahramanmaraş, Turkey \\ ${ }^{2}$ Ankara University, Faculty of Science, Department of Mathematics, 06100, Ankara, Turkey
}

\section{Highlights}

- This paper focuses on the special curves.

- This study is a polynomial approach to rectifying curves.

- This study is important because it is an application of differential equations and curves.

\section{Article Info}

Received:27/02/2020

Accepted: 05/09/2020

\section{Keywords}

Rectifying curve

Salkowski curve

Harmonic curvature

\begin{abstract}
The aim of the paper is to find polynomial parametric equations of rectifying Salkowski curves in Minkowski 3-space, via a serial approach. These curves are characterized by according to their curvature; in particular those curves with constant curvature functions and linear harmonic curvature functions are fully characterized. Then, the equations of the rectifying Salkowski curves are obtained as serial solutions of differential equations with third-order polynomial coefficients.
\end{abstract}

\section{INTRODUCTION}

Curves are one of the important fields of study in differential geometry. Because there are many applications in the real world, curves such as helices, slant helices, Bertrand, isophote curves, etc. are the most important for curves theory. One of these types of curves is rectifying curves. Rectifying curves are introduced by B. Y. Chen in [1]. The position vectors of these curves always lie in their rectifying planes. More clearly, the position vector of a rectifying curve $\alpha$ is shown as

$$
\alpha(s)=\lambda(s) T(s)+\mu(s) B(s),
$$

for some arbitrary differentiable functions $\lambda$ and $\mu$. One of the most interesting characteristic features of rectifying curves is that its harmonic curvature function is a non-constant linear function according to the arclength parameter $s$. In addition, B. Y. Chen obtained some different results about rectifying curves in [2]. Rectifying curves in Minkowski 3-space have a similar geometric properties as in Euclidean 3space. For instance, the ratio of torsion to curvature of any regular rectifying curve in $E_{1}^{3}$ is non-constant linear function with respect to parameter $s$ [3].

Salkowski curves are generally known as curves with constant curvature, but non-constant torsion. These curves are studied by many authors such as in [4], [5] etc. Y1lmaz et.al. studied rectifying salkowski/anti salkowski curves in Galilean 3-space [6].

Let $\gamma: I \subset R \rightarrow E^{3}$ be a unit speed curve with Frenet frame apparatus $\{T, N, B, \kappa, \tau\}$. The ratio of the torsion to the curvature of the curve $\gamma$ is defined as the harmonic curvature function of $\gamma$. This curvature is indicated by the notation $H=\frac{\tau}{\kappa}$ and is defined by Özdamar and Hacisalihoğlu [7]. 
In this study, we define the curves in Minkowski 3-space with constant curvature function and linear harmonic curvature function. These types of curves are studied in Euclidean 3-space by Oh and Seo [8]. If the harmonic curvature function of the curve is linear, we know that this curve is called rectifying curve. Also, if the curvature of the curve is positive constant, we know that this curve is called Salkowski curve. So, we called these curves as rectifying Salkowski curves. The purpose of this study is to find a clear formula of the rectifying Salkowski curves with the help of the serial solutions of differential equations with third-order polynomial coefficients.

\section{PRELIMINARIES}

Let $E_{1}^{3}$ be Minkowski 3-space with the following metric

$\langle,\rangle_{L}=R^{3} \times R^{3} \rightarrow R$

$(u, v) \rightarrow\langle u, v\rangle_{L}=u_{1} v_{1}+u_{2} v_{2}+u_{3} v_{3}$,

where $u=\left(u_{1}, u_{2}, u_{3}\right)$ and $v=\left(v_{1}, v_{2}, v_{3}\right)$ are the usual coordinate system in $E_{1}^{3}$. An arbitrary vector $u \in E_{1}^{3}$ is named spacelike if $\langle u, u\rangle_{L}>0$ or $u=0$, timelike if $\langle u, u\rangle_{L}<0$ and null (lightlike) if $\langle u, u\rangle_{L}=0$ but $u \neq 0$. The norm of a vector $u$ is given by $\|u\|_{L}=\sqrt{\left|\langle u, u\rangle_{L}\right|}$.

Let $\{T(s), N(s), B(s)\}$ be the Frenet frame in Minkowski 3-space along the unit speed curve $\gamma$. Here $T(s)=\gamma^{\prime}(s)$ is a tangent vector, $N(s)=\frac{\gamma^{\prime \prime}(s)}{\left\|\gamma^{\prime \prime}(s)\right\|}$ is a principal normal vector and $B(s)=T(s) \times N(s)$ is a binormal vector field along the curve $\gamma$ [9].

If the Frenet vectors of $\gamma$ in Minkowski 3-space satisfy following equalities,

$$
\begin{aligned}
& \langle T(s), T(s)\rangle_{L}=\varepsilon_{1},\langle N(s), N(s)\rangle_{L}=\varepsilon_{2},\langle B(s), B(s)\rangle_{L}=\varepsilon_{3}, \\
& \langle T(s), N(s)\rangle_{L}=\langle T(s), B(s)\rangle_{L}=\langle N(s), B(s)\rangle_{L}=0,
\end{aligned}
$$

then Frenet formulas are given as follows

$$
\left(\begin{array}{c}
T^{\prime}(s) \\
N^{\prime}(s) \\
B^{\prime}(s)
\end{array}\right)=\left(\begin{array}{ccc}
0 & \kappa(s) & 0 \\
-\varepsilon_{1} \varepsilon_{2} \kappa(s) & 0 & -\varepsilon_{2} \varepsilon_{3} \tau(s) \\
0 & \tau(s) & 0
\end{array}\right)\left(\begin{array}{c}
T(s) \\
N(s) \\
B(s)
\end{array}\right),
$$

where $\kappa(s)$ and $\tau(s)$ are curvature and torsion of $\gamma$, respectively [10].

\section{POLYNOMIAL PARAMETRIC EQUATIONS OF RECTIFYING SALKOWSKI CURVES}

In this section, curves with constant curvature and non-zero linear harmonic curvature are investigated in Minkowski 3-space. In other words, the polynomial equation of rectifying curves is obtained with the condition that the curvature function is constant. To obtain this equation, the serial approach for polynomial coefficients differential equation of principal normal vector of the curve $\gamma$ is used.

Let $\kappa(s)=\left|T^{\prime}(s)\right|>0$ be a constant and $H(s)$ be a non-zero linear, that is, $H=\frac{\tau}{\kappa}=s$ or $\tau=\kappa s$. If the new parameterization $t$ of the curve $\gamma$ is denoted by 
$t(s)=\int_{0}^{s} \kappa d \sigma=\kappa s$

we can reconstruct the Serret-Frenet formulas with the new parameter as follows

$\frac{d T}{d t}=\frac{d T}{d s} \frac{d s}{d t}=N$

$\frac{d N}{d t}=\frac{d N}{d s} \frac{d s}{d t}=-\varepsilon_{1} \varepsilon_{2} T-\varepsilon_{2} \varepsilon_{3} \frac{t}{\kappa} B$

$\frac{d B}{d t}=\frac{d B}{d s} \frac{d s}{d t}=\frac{t}{\kappa} N$

If we take the derivative of the principal vector $N$ of the curve $\gamma$, we get

$$
\begin{aligned}
& \frac{d^{2} N}{d t^{2}}=-\varepsilon_{1} \varepsilon_{2} \frac{d T}{d t}-\varepsilon_{2} \varepsilon_{3} \frac{1}{\kappa} B-\varepsilon_{2} \varepsilon_{3} \frac{t}{\kappa} \frac{d B}{d t}, \\
& =-\varepsilon_{1} \varepsilon_{2} N-\varepsilon_{2} \varepsilon_{3} \frac{1}{\kappa} B-\varepsilon_{2} \varepsilon_{3} \frac{t^{2}}{\kappa^{2}} N
\end{aligned}
$$

and then

$$
\begin{aligned}
& \frac{d^{3} N}{d t^{3}}=-\varepsilon_{1} \varepsilon_{2} \frac{d N}{d t}-\varepsilon_{2} \varepsilon_{3} \frac{1}{\kappa} \frac{d B}{d t}-\varepsilon_{2} \varepsilon_{3} \frac{2 t}{\kappa^{2}} N-\varepsilon_{2} \varepsilon_{3} \frac{t^{2}}{\kappa^{2}} \frac{d N}{d t}, \\
& =-\varepsilon_{1} \varepsilon_{2} \frac{d N}{d t}-\varepsilon_{2} \varepsilon_{3} \frac{t}{\kappa^{2}} N-\varepsilon_{2} \varepsilon_{3} \frac{2 t}{\kappa^{2}} N-\varepsilon_{2} \varepsilon_{3} \frac{t^{2}}{\kappa^{2}} \frac{d N}{d t}, \\
& =-\left(\varepsilon_{1} \varepsilon_{2}+\varepsilon_{2} \varepsilon_{3} \frac{t^{2}}{\kappa^{2}}\right) \frac{d N}{d t}-\varepsilon_{2} \varepsilon_{3} \frac{3 t}{\kappa^{2}} N .
\end{aligned}
$$

So, if necessary arrangements are made in above equalities, third-order differential equation of $N=N(t)$ is obtained as

$$
\frac{d^{3} N}{d t^{3}}+\left(\varepsilon_{1} \varepsilon_{2}+\varepsilon_{2} \varepsilon_{3} \frac{t^{2}}{\kappa^{2}}\right) \frac{d N}{d t}+\varepsilon_{2} \varepsilon_{3} \frac{3 t}{\kappa^{2}} N=0
$$

Since $t=0$ is an ordinary point for this linear differential equation, there exists a serial solution in a neighborhood of $t=0$, that is, the principal normal vector of $\gamma$ can be given with coefficient vectors $a_{n}$ as follows

$$
N=\sum_{n=0}^{\infty} a_{n} t^{n}
$$

In the Equation (2), if we consider this serial approach, we have

$$
\sum_{n=3}^{\infty} n(n-1)(n-2) a_{n} t^{n-3}+\left(\varepsilon_{1} \varepsilon_{2}+\varepsilon_{2} \varepsilon_{3} \frac{t^{2}}{\kappa^{2}}\right) \sum_{n=1}^{\infty} n a_{n} t^{n-1}+\varepsilon_{2} \varepsilon_{3} \frac{3 t}{\kappa^{2}} \sum_{n=0}^{\infty} a_{n} t^{n}=0
$$

and

$$
\sum_{n=0}^{\infty}(n+3)(n+2)(n+1) a_{n+3} t^{n}+\sum_{n=0}^{\infty} \varepsilon_{1} \varepsilon_{2}(n+1) a_{n+1} t^{n}+\sum_{n=2}^{\infty} \frac{\varepsilon_{2} \varepsilon_{3}}{\kappa^{2}}(n-1) a_{n-1} t^{n}+\sum_{n=1}^{\infty} \frac{3 \varepsilon_{2} \varepsilon_{3}}{\kappa^{2}} a_{n-1} t^{n}=0 .
$$


Using the values $n=0$ and $n=1$, the equation can be edited as

$$
\begin{aligned}
& \left(\varepsilon_{1} \varepsilon_{2} a_{1}+6 a_{3}\right)+\left(\frac{3 \varepsilon_{2} \varepsilon_{3}}{\kappa^{2}} a_{0}+2 \varepsilon_{1} \varepsilon_{2} a_{2}+24 a_{4}\right) t+\sum_{n=2}^{\infty}\left((n+3)(n+2)(n+1) a_{n+3}\right) t^{n} \\
& +\sum_{n=2}^{\infty}\left(\varepsilon_{1} \varepsilon_{2}(n+1) a_{n+1}+\frac{\varepsilon_{2} \varepsilon_{3}}{\kappa^{2}}(n-1) a_{n-1}+\frac{3 \varepsilon_{2} \varepsilon_{3}}{\kappa^{2}} a_{n-1}\right) t^{n}=0 .
\end{aligned}
$$

Then, coefficients $a_{3}$ and $a_{4}$ are obtained

$a_{3}=-\frac{\varepsilon_{1} \varepsilon_{2}}{6} a_{1}$ and $a_{4}=-\frac{\varepsilon_{1} \varepsilon_{2}}{12} a_{2}-\frac{\varepsilon_{2} \varepsilon_{3}}{8 \kappa^{2}} a_{0}$.

Moreover, if Equation (3) for $n=2$ and Equation (4) are considered together, we find

$$
a_{5}=\left(\frac{1}{120}-\frac{\varepsilon_{2} \varepsilon_{3}}{15 \kappa^{2}}\right) a_{1} \text {. }
$$

For $n=3$ in the Equation (3), we have

$$
120 a_{6}+4 \varepsilon_{1} \varepsilon_{2} a_{4}+\frac{5 \varepsilon_{2} \varepsilon_{3}}{\kappa^{2}} a_{2}=0
$$

or using Equation (4), we obtain that

$$
a_{6}=\left(-\frac{\varepsilon_{2} \varepsilon_{3}}{24 \kappa^{2}}+\frac{1}{360}\right) a_{2}+\frac{\varepsilon_{1} \varepsilon_{3}}{240 \kappa^{2}} a_{0} .
$$

Similarly, if we continue this process, then we determine the coefficients of the principal normal.

$$
\begin{aligned}
& N(t)=a_{0}+a_{1} t+a_{2} t^{2}+\left(-\frac{\varepsilon_{1} \varepsilon_{2}}{6} a_{1}\right) t^{3}+\left(-\frac{\varepsilon_{1} \varepsilon_{2}}{12} a_{2}-\frac{\varepsilon_{2} \varepsilon_{3}}{8 \kappa^{2}} a_{0}\right) t^{4} \\
& +\left(\frac{1}{120}-\frac{\varepsilon_{2} \varepsilon_{3}}{15 \kappa^{2}}\right) a_{1} t^{5}+\left[\left(\frac{1}{360}-\frac{\varepsilon_{2} \varepsilon_{3}}{24 \kappa^{2}}\right) a_{2}+\frac{\varepsilon_{1} \varepsilon_{3}}{240 \kappa^{2}} a_{0}\right] t^{6}+\ldots
\end{aligned}
$$

Regulating the Equation (7) according to its coefficients, we can easily see that

$$
\begin{aligned}
& N(t)=a_{0}\left[1-\frac{\varepsilon_{2} \varepsilon_{3}}{8 \kappa^{2}} t^{4}+\frac{\varepsilon_{1} \varepsilon_{3}}{240 \kappa^{2}} t^{6}+\left(\frac{1}{384 \kappa^{2}}-\frac{\varepsilon_{2} \varepsilon_{3}}{13440 \kappa^{2}}\right) t^{8}+\ldots\right] \\
& +a_{1}\left[t-\frac{\varepsilon_{1} \varepsilon_{2}}{6} t^{3}+\left(\frac{1}{120}-\frac{\varepsilon_{2} \varepsilon_{3}}{15 \kappa^{2}}\right) t^{5}+\left(-\frac{\varepsilon_{1} \varepsilon_{2}}{5040}+\frac{2 \varepsilon_{1} \varepsilon_{3}}{315 \kappa^{2}}\right) t^{7}+\ldots\right] \\
& +a_{2}\left[t^{2}-\frac{\varepsilon_{1} \varepsilon_{2}}{12} t^{4}+\left(\frac{1}{360}-\frac{\varepsilon_{2} \varepsilon_{3}}{24 \kappa^{2}}\right) t^{6}+\left(-\frac{\varepsilon_{1} \varepsilon_{2}}{20160}+\frac{5 \varepsilon_{1} \varepsilon_{3}}{2016 \kappa^{2}}\right) t^{8}+\ldots\right] .
\end{aligned}
$$

To find the tangent vector $T$, we need to integrate the above equation. So, it gives us the tangent vector as follows 


$$
\begin{aligned}
& T(t)=a_{0}\left[t-\frac{\varepsilon_{2} \varepsilon_{3}}{40 \kappa^{2}} t^{5}+\frac{\varepsilon_{1} \varepsilon_{3}}{1680 \kappa^{2}} t^{7}+\left(\frac{1}{3456 \kappa^{2}}-\frac{\varepsilon_{2} \varepsilon_{3}}{120960 \kappa^{2}}\right) t^{9}+\ldots\right] \\
& +a_{1}\left[\frac{t^{2}}{2}-\frac{\varepsilon_{1} \varepsilon_{2}}{24} t^{4}+\left(\frac{1}{720}-\frac{\varepsilon_{2} \varepsilon_{3}}{90 \kappa^{2}}\right) t^{6}+\left(-\frac{\varepsilon_{1} \varepsilon_{2}}{40320}+\frac{\varepsilon_{1} \varepsilon_{3}}{1260 \kappa^{2}}\right) t^{8}+\ldots\right] \\
& +a_{2}\left[\frac{t^{3}}{3}-\frac{\varepsilon_{1} \varepsilon_{2}}{60} t^{5}+\left(\frac{1}{2520}-\frac{\varepsilon_{2} \varepsilon_{3}}{168 \kappa^{2}}\right) t^{7}+\left(-\frac{\varepsilon_{1} \varepsilon_{2}}{181440}+\frac{5 \varepsilon_{1} \varepsilon_{3}}{18144 \kappa^{2}}\right) t^{9}+\ldots\right] \\
& +b
\end{aligned}
$$

where $a_{0}, a_{1}, a_{2}$ and $b$ are constant vectors.

Since $\gamma^{\prime}(t)=T$, to find the equation of the curve $\gamma$, we integrate the above equation according to the parameter $t$. Consequently, the polynomial equation of the curve $\gamma$ with constant curvature function and linear harmonic curvature function is obtained by

$$
\begin{aligned}
& \gamma(t)=a_{0}\left[\frac{t^{2}}{2}-\frac{\varepsilon_{2} \varepsilon_{3}}{240 \kappa^{2}} t^{6}+\frac{\varepsilon_{1} \varepsilon_{3}}{13440 \kappa^{2}} t^{8}+\left(\frac{1}{34560 \kappa^{2}}-\frac{\varepsilon_{2} \varepsilon_{3}}{1209600 \kappa^{2}}\right) t^{10}+\ldots\right] \\
& +a_{1}\left[\frac{t^{3}}{3}-\frac{\varepsilon_{1} \varepsilon_{2}}{120} t^{5}+\left(\frac{1}{5040}-\frac{\varepsilon_{2} \varepsilon_{3}}{630 \kappa^{2}}\right) t^{7}+\left(-\frac{\varepsilon_{1} \varepsilon_{2}}{362880}+\frac{\varepsilon_{1} \varepsilon_{3}}{11340 \kappa^{2}}\right) t^{9}+\ldots\right] \\
& +a_{2}\left[\frac{t^{4}}{12}-\frac{\varepsilon_{1} \varepsilon_{2}}{360} t^{6}+\left(\frac{1}{20160}-\frac{\varepsilon_{2} \varepsilon_{3}}{1344 \kappa^{2}}\right) t^{8}+\left(-\frac{\varepsilon_{1} \varepsilon_{2}}{1814400}+\frac{\varepsilon_{1} \varepsilon_{3}}{36288 \kappa^{2}}\right) t^{10}+\ldots\right] \\
& +b t+c
\end{aligned}
$$

for $a_{0}, a_{1}, a_{2}, b$ and $c$ are constant vectors in $E_{1}^{3}$.

\subsection{Construction of Rectifying Salkowski Curves with Initial Conditions}

In this subsection, the unknown constant vectors $a_{0}, a_{1}, a_{2}, b$ and $c$ are analyzed with the help of some initial conditions. The curve $\gamma(t)$ is exactly determined with the help of them. We would like to emphasize clearly that rectifying Salkowski curves can be constructed depending on different initial conditions. Depending on the following condition, the serial parametric representation of a rectifying Salkowski curves are obtained in Corollary 1. Moreover, an example of the curve created with the same initial conditions is also given.

Assume that $\gamma(0)=c=(0,0,0)$ and $T(0)=b=(0,1,0)$, that is, $c=0$ and $b=j$. If we suppose $a_{0}=i=(1,0,0)$ without loss of generality, we can give an initial condition as $N(0)=a_{0}$. Using the Equation (1) and $N^{\prime}(0)=a_{1}$, we find the following equation

$$
N^{\prime}(t)=-\varepsilon_{1} \varepsilon_{2} T(t)-\varepsilon_{2} \varepsilon_{3} \frac{t}{\kappa} B(t)
$$

For $t=0$,

$$
N^{\prime}(0)=-\varepsilon_{1} \varepsilon_{2} T(0)=a_{1}=-\varepsilon_{1} \varepsilon_{2} j
$$

the coefficient $a_{1}$ is as follows

$a_{1}=-\varepsilon_{1} \varepsilon_{2} j$. 
If we calculate the second order derivative of $N(t)$ for $t=0$, we can easily see that

$N^{\prime \prime}(0)=2 a_{2}$.

On the other hand, we can easily see from Equation (1)

$N^{\prime \prime}(t)=-\varepsilon_{1} \varepsilon_{2} N(t)-\varepsilon_{2} \varepsilon_{3} \frac{1}{\kappa} B(t)-\varepsilon_{2} \varepsilon_{3} \frac{t^{2}}{\kappa^{2}} N(t)$.

For $t=0$,

$N^{\prime \prime}(0)=-\varepsilon_{1} \varepsilon_{2} N(0)-\varepsilon_{2} \varepsilon_{3} \frac{1}{\kappa} B(0)$.

Using the equality $B(0)=T(0) \times N(0)=j \times i=k$, we get

$N^{\prime \prime}(0)=-\varepsilon_{1} \varepsilon_{2} i-\varepsilon_{2} \varepsilon_{3} \frac{1}{\kappa} k$.

If the Equations (9) and (10) are considered with together, we can easily obtain that

$a_{2}=\frac{1}{2}\left(-\varepsilon_{1} \varepsilon_{2}, 0,-\varepsilon_{2} \varepsilon_{3} \frac{1}{\kappa}\right)$.

Thus, coefficient vectors $a_{0}, a_{1}, a_{2}, b$ and $c$ are as follows

$a_{0}=(1,0,0), a_{1}=\left(0,-\varepsilon_{1} \varepsilon_{2}, 0\right), a_{2}=\left(-\frac{\varepsilon_{1} \varepsilon_{2}}{2}, 0, \frac{\varepsilon_{2} \varepsilon_{3}}{2 \kappa}\right), b=(0,1,0), c=(0,0,0)$.

So, the equation of the curve $\gamma$ obtained by the above initial conditions is expressed in the following corollary.

Corollary 1. Let $\gamma: I \subset R \rightarrow E_{1}^{3}$ be a unit speed curve with constant curvature function $\kappa>0$ and linear harmonic curvature function $H$. Then the reparametrization of the curve $\gamma$ is

$$
\gamma(t)=\left(f_{1}(t)-\frac{\varepsilon_{1} \varepsilon_{2}}{2} f_{3}(t),-\varepsilon_{1} \varepsilon_{3} f_{2}(t)+t,-\frac{\varepsilon_{2} \varepsilon_{3}}{2 \kappa} f_{3}(t)\right),
$$

where

$$
\begin{aligned}
& f_{1}(t)=\frac{t^{2}}{2}-\frac{\varepsilon_{2} \varepsilon_{3}}{240 \kappa^{2}} t^{6}+\frac{\varepsilon_{1} \varepsilon_{3}}{13440 \kappa^{2}} t^{8}+\left(\frac{1}{34560 \kappa^{2}}-\frac{\varepsilon_{2} \varepsilon_{3}}{1209600 \kappa^{2}}\right) t^{10}+\ldots \\
& f_{2}(t)=\frac{t^{3}}{3}-\frac{\varepsilon_{1} \varepsilon_{2}}{120} t^{5}+\left(\frac{1}{5040}-\frac{\varepsilon_{2} \varepsilon_{3}}{630 \kappa^{2}}\right) t^{7}+\left(-\frac{\varepsilon_{1} \varepsilon_{2}}{362880}+\frac{\varepsilon_{1} \varepsilon_{3}}{11340 \kappa^{2}}\right) t^{9}+\ldots \\
& f_{3}(t)=\frac{t^{4}}{12}-\frac{\varepsilon_{1} \varepsilon_{2}}{360} t^{6}+\left(\frac{1}{20160}-\frac{\varepsilon_{2} \varepsilon_{3}}{1344 \kappa^{2}}\right) t^{8}+\left(-\frac{\varepsilon_{1} \varepsilon_{2}}{1814400}+\frac{\varepsilon_{1} \varepsilon_{3}}{36288 \kappa^{2}}\right) t^{10}+\ldots
\end{aligned}
$$

Example 1. Let $\gamma: I \subset R \rightarrow E_{1}^{3}$ be a unit speed timelike curve in Minkowski 3-space. Furthermore, the initial conditions be $T(0)=b=(0,1,0)=j$ and $c=(0,0,0)$. If we assume that $\varepsilon_{1}=-1, \varepsilon_{2}=1, \varepsilon_{3}=1$ and $N(0)=a_{0}=(1,0,0)=i$, similar operations in the preceding section gives us $N^{\prime}(0)=a_{1}=-\varepsilon_{1} \varepsilon_{2} T(0)$ and $a_{1}=(0,1,0)=j$. Finally, using $N^{\prime \prime}(0)=2 a_{2}=-\varepsilon_{1} \varepsilon_{2} N(0)-\varepsilon_{2} \varepsilon_{3} \frac{1}{\kappa} B(0)$ and $B(0)=T(0) \times N(0)=k$, we obtain $a_{2}=\left(\frac{1}{2}, 0,-\frac{1}{2 \kappa}\right)$. 
Thus, all coefficient vectors are obtained as follows

$$
a_{0}=(1,0,0), a_{1}=(0,1,0), a_{2}=\left(\frac{1}{2}, 0,-\frac{1}{2 \kappa}\right), b=(0,1,0), c=(0,0,0) .
$$

So, the curve $\gamma$ is obtained as

$$
\begin{aligned}
& \gamma(t)=\left(\frac{t^{2}}{2}-\frac{t^{6}}{240 \kappa^{2}}-\frac{t^{8}}{13440 \kappa^{2}}+\left(\frac{1}{34560 \kappa^{2}}-\frac{1}{1209600 \kappa^{2}}\right) t^{10}+\ldots\right. \\
& +\frac{t^{4}}{24}+\frac{t^{6}}{720}+\left(\frac{1}{40320}-\frac{1}{2688 \kappa^{2}}\right) t^{8}+\left(\frac{1}{3628800}-\frac{1}{72576 \kappa^{2}}\right) t^{10}+\ldots, \\
& \frac{t^{3}}{3}+\frac{t^{5}}{120}+\left(\frac{1}{5040}-\frac{1}{630 \kappa^{2}}\right) t^{7}+\left(\frac{1}{362880}-\frac{1}{11340 \kappa^{2}}\right) t^{9}+t \ldots, \\
& \left.-\frac{t^{4}}{24 \kappa}-\frac{t^{6}}{720 \kappa}+\left(-\frac{1}{40320 \kappa}+\frac{1}{2688 \kappa^{3}}\right) t^{8}+\left(-\frac{1}{3628800 \kappa}+\frac{1}{72576 \kappa^{3}}\right) t^{10}+\ldots\right) .
\end{aligned}
$$

Since the curve $\gamma(t)$ has a constant curvature, we can get $\kappa=1$. Thus, the figure of $\gamma$ is drawn by using Mathematica programme (Figure 1)

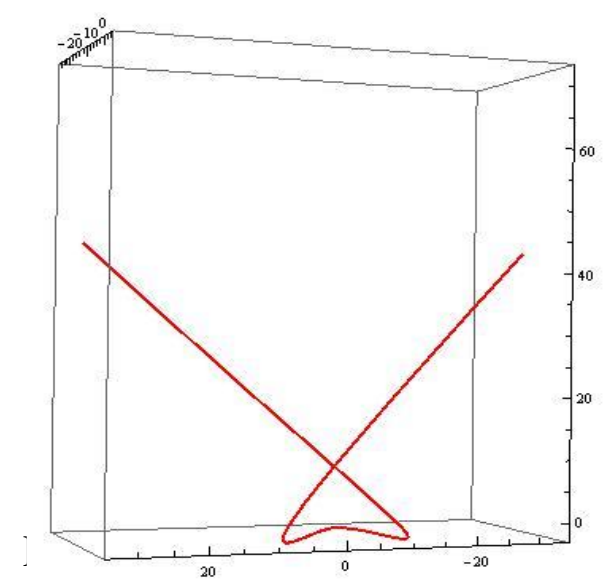

Figure 1. Timelike Rectifying Salkowski Curve $\gamma(t)$

In the following example, the rectifying Salkowski curve is constructed in terms of some different initial conditions.

Example 2. Let $\gamma: I \subset R \rightarrow E_{1}^{3}$ be a unit speed spacelike curve in Minkowski 3-space. Assume that $\varepsilon_{1}=1, \varepsilon_{2}=1, \varepsilon_{3}=-1$ and $T(0)=b=(1,0,0)=i, N(0)=a_{0}=(0,0,1)=k$ and $c=(0,0,0)$. So, $N^{\prime}(0)=a_{1}=\left(-\varepsilon_{1} \varepsilon_{2}, 0,0\right), N^{\prime \prime}(0)=2 a_{2}=-\varepsilon_{1} \varepsilon_{2} N(0)-\varepsilon_{2} \varepsilon_{3} \frac{1}{\kappa} B(0) \quad$ and $\quad B(0)=j$ are obtained. If necessary arrangements are made, then we have $a_{2}=\left(0, \frac{\varepsilon_{2} \varepsilon_{3}}{2 \kappa},-\frac{\varepsilon_{1} \varepsilon_{2}}{2}\right)$. Thus, all the coefficient vectors are given as follows

$a_{0}=(0,0,1), a_{1}=(-1,0,0), a_{2}=\left(0, \frac{1}{2 \kappa},-\frac{1}{2}\right), b=(1,0,0), c=(0,0,0)$.

So, the curve $\gamma$ is found as 


$$
\begin{aligned}
& \gamma(t)=\left(-\frac{t^{3}}{3}+\frac{t^{5}}{120}-\left(\frac{1}{5040}+\frac{1}{630 \kappa^{2}}\right) t^{7}+\left(\frac{1}{362880}+\frac{1}{11340 \kappa^{2}}\right) t^{9}+t \ldots\right. \\
& \frac{t^{4}}{24 \kappa}-\frac{t^{6}}{720 \kappa}+\left(\frac{1}{40320 \kappa}+\frac{1}{2688 \kappa^{3}}\right) t^{8}-\left(\frac{1}{3628800 \kappa}+\frac{1}{72576 \kappa^{3}}\right) t^{10}+\ldots, \\
& \frac{t^{2}}{2}+\frac{t^{6}}{240 \kappa^{2}}-\frac{t^{8}}{13440 \kappa^{2}}+\left(\frac{1}{34560 \kappa^{2}}+\frac{1}{1209600 \kappa^{2}}\right) t^{10}+\ldots \\
& -\frac{t^{4}}{24}+\frac{t^{6}}{720}-\left(\frac{1}{40320}+\frac{1}{2688 \kappa^{2}}\right) t^{8}+\left(\frac{1}{3628800}+\frac{1}{72576 \kappa^{2}}\right) t^{10}+\ldots
\end{aligned}
$$

Since the curve $\gamma(t)$ has a constant curvature, we can get $\kappa=1$. Thus, the figure of $\gamma$ is drawn by using Mathematica programme (Figure 2)

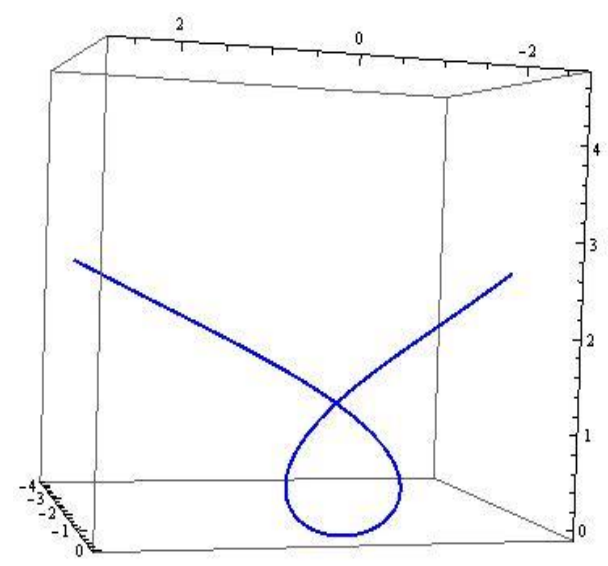

Figure 2. Spacelike Rectifying Salkowski Curve $\gamma(t)$

\section{CONFLICTS OF INTEREST}

No conflict of interest was declared by the authors.

\section{REFERENCES}

[1] Chen, B.Y., "When does the position vector of a space curve always lie in its rectifying plane?", Amer. Math. Monthly, 110: 147-152, (2003).

[2] Chen, B.Y., "Topics in differential geometry associated with position vector fields on Euclidean submanifolds", Arab Journal of Mathematical Sciences, 23: 1-17, (2017).

[3] İlarslan, K., Nesovic, E., Petrovic-Torgasev, M., "Some characterizations of rectifying curves in the Minkowski 3-space", Novi Sad Journal of Mathematics, 33(2): 23-32, (2003).

[4] Monterde, J., "Salkowski curves revisited, A family of curves with constant curvature and nonconstant torsion", Computer Aided Geometric Design, 26: 271-278, (2009).

[5] Salkowski, E.E., "Zur transformation von raumkurven”, Mathematische Annalen, 66(4): 517557, (1909). 
[6] Yılmaz, B., Metin, Ş., Gök, İ. and Yaylı, Y., "Harmonic curvature functions of some special curves in Galilean 3-space", Honam Mathematical Journal, 41(2): 301-309, (2019).

[7] Özdamar, E., Hacisalihoğlu, H.H., "A characterization of inclined curves in Euclidean n-space", Communication de la facult'e des sciences de L'Universit'e d'Ankara, 24: 15-22, (1975).

[8] Oh, Y.M., Seo, Y.L., "A Curve Satisfying $\tau / \kappa=s$ with constant $\kappa>0$ ”, American Journal of Undergraduate Research, 2(12): 57-62, (2015).

[9] Do Carmo, M., "Differential geometry of curves and surfaces", Prentice-Hall, Upper Saddle Riv. N.J., (1976).

[10] O'Neill, B., "Semi-Riemannian Geometry with Application to Relativity", Academic Press, New York, (1983). 